\title{
Correction to: A higher Boltzmann distribution
}

\author{
Michael J. Catanzaro ${ }^{1}$ - Vladimir Y. Chernyak ${ }^{2}$. John R. Klein ${ }^{3}$
}

Published online: 24 July 2021

(c) Springer Nature Switzerland AG 2021

\section{Correction to: J Appl. and Comput. Topology https://doi.org/10.1007/s41468-017-0006-9}

The original publication of the article Catanzaro et al. (2017) contained the following minor errors which we take this opportunity to correct:

- In Remark 1.11:

The statement, "Conditions (a)-(c) are equivalent to conditions (a'), (b) and (c)" should be replaced by "Conditions (a)-(c) imply conditions (a'), (b) and (c)"

- In Remark 2.7:

The sentence beginning with, "Let $T_{d-1}(X ; \mathbb{Z}) \subset \ldots$ " until the end of the remark should be removed.

- In the last paragraph of the proof of Theorem A:

The text

"....and $\phi_{L}$ corresponds to $A_{S}$. Then the determinant of $A_{S}$ is given up to sign by $\tau_{L}^{\prime}=\tau_{L} / \theta_{X}$ (cf. Remark 2.7) and the prefactor is given by the reciprocal of $\nabla^{\prime}:=\sum_{L} \tau_{L}^{\prime}$. Hence, the desired splitting is given by

$$
\frac{1}{\nabla^{\prime}} \sum \tau_{L}^{\prime} \psi_{L}=\frac{1}{\nabla} \sum \tau_{L} \psi_{L} .
$$

should be replaced by the text

“....and $A_{S}$ corresponds to $C_{d-1}(L) \rightarrow C_{d-1}(X) / B_{d-1}(X)$. Then the determinant

The original article can be found online at https://doi.org/10.1007/s41468-017-0006-9.

John R. Klein

klein@math.wayne.edu

Michael J. Catanzaro

mjcatanz@iastate.edu

Vladimir Y. Chernyak

chernyak@chem.wayne.edu

1 Department of Mathematics, Iowa State University, Ames, IA 50011, USA

2 Department of Chemistry, Wayne State University, Detroit, MI 48202, USA

3 Department of Mathematics, Wayne State University, Detroit, MI 48202, USA 
of $A_{S}$ is given up to sign by $a_{L}$ (cf. Remark 2.7), and $t_{S}=\tau_{L}$. Hence, the desired splitting is given by

$$
\frac{1}{\nabla} \sum \tau_{L} \psi_{L}
$$

- In Theorem 3.3, the text:

"of rank $m \mathrm{O} \mathbb{R}$ "

should be replaced by the text

"of rank $m$ defined over $\mathbb{R}$ "

\section{References}

Catanzaro, Michael J., Chernyak, Vladimir Y., Klein, John R.: A higher Boltzmann distribution. J. Appl. Comput. Topol. 1(2), 215-240 (2017). https://doi.org/10.1007/s41468-017-0006-9

Publisher's Note Springer Nature remains neutral with regard to jurisdictional claims in published maps and institutional affiliations. 